\title{
ON A STEP METHOD AND A PROPAGATION OF DISCONTINUITY
}

\author{
DIANA CAPONETTI, MIECZYSŁAW CICHOŃ, AND VALERIA MARRAFFA
}

\begin{abstract}
In this paper we analyze how to compute discontinuous solutions for functionaldifferential equations, looking at an approach which allows to study simultaneously continuous and discontinuous solutions. We focus our attention on the integral representation of solutions and we justify the applicability of such an approach. In particular, we improve the step method in such a way to solve a problem of vanishing discontinuity points. Our solutions are considered as regulated functions.
\end{abstract}

\section{InTRODUCTION.}

In the classical theory of differential or integral equations generally solutions are expected to be at least continuous. However, for many types of delay-differential equations (DDE's), for impulsive equations or measure differential equations, solutions need not be continuous (cf. [25, Chapter 2.5.2], [41, Chapter 5] or [39]). Despite that the problem can be also considered in terms of distributional derivative (see, for instance, [16]) we believe that it is too far from the problems leading to DDE's and in this paper we keep the advantages of the classical theory. All derivatives considered here are taken in the classical sense.

In the paper we concentrate on computational aspects of this theory, so we study the applicability of the step method for discontinuous initial functions and then for problems having discontinuous solutions. There are two main problems to be solved: what kind of integral representations of solutions can be applied for the step method and how to ensure the propagation of discontinuity points from the initial interval to the future. Our results are of practical nature and they should allow the use of mathematical models based on DDE's with discontinuous initial functions with the "almost classical" step method.

Motivated by the papers $[4,5]$ we will study the problem of discontinuous solutions for DDE's. In this case, the definition and the construction of solutions seem to be rather complicated with respect to the case of ordinary differential equations (ODE's). Here we suggest a simpler approach which allows to use the same types of proof for both cases. We consider some retarded functionaldifferential equation of the form

$$
\begin{aligned}
x^{\prime}(t) & =f(t, x(t-\tau)) \\
x(t) & =\phi(t) \quad \text { for } t \in[-\tau, 0] .
\end{aligned}
$$

Date: October, 29, 2018

1991 Mathematics Subject Classification. 34K05, 34K40.

Key words and phrases. regulated function, discontinuous function, retarded differential equation, delay, Kurzweil-Stieltjes integral, breaking points.

The second author was partially supported by INdAM-GNAMPA n. prot. U 2016/000386. 
Problems of this kind arise, for instance, in population dynamics (such as the growth of cell modelling: $x^{\prime}(t)=\frac{1}{\tau} x(t-\tau)$, see [6,26]; with discontinuous initial function $\phi$, see [3, Section 1.4] for more details).

Let us stress that, in view of seasonability of some population dynamics, the population observed as discontinuous in the past should be discontinuous in the future and the model should preserve this property, we recall that time scales are sometimes used to describe such a situation (cf. [10], for instance). As in this case we are able to think that $\phi$ has values zero outside of the time scale (whence it is possibly discontinuous), we cover and extend this approach too. Thus both continuous and discontinuous functions should be considered as solutions and we unify both cases in one approach. The choice of the study of the problem (1) is sufficient to show all the ideas which will be then applied for more general problems.

The classical theory for DDE's is based on the assumption that the initial function $\phi$ is continuous on $[-\tau, 0]$, consequently solutions are continuous too. As claimed above, in some mathematical models, it is not a realistic case. Following [5] we will allow the function $\phi$ to be discontinuous at some points in $[-\tau, 0]$. As $x$ is determined by the initial function $\phi$ the solution is expected to be discontinuous too.

We think that regulated functions constitute a convenient function space with respect to the supremum-norm to study problems of this form. Due to some properties of the space of regulated functions we will be able to extend the results to this space in a manner unified with continuous case. We will give a proper integral representation for problem (1) defined in terms of Stieltjes-type integrals, namely using the Kurzweil-Stieltjes integral. Note that integral equations with Kurzweiltype integrals were investigated (see [16], for instance) mainly by its generality. It is known, that not only DDE's, but also some other functional equations can be treated as special cases for generalized differential equations and the existence theorems are then proved (cf. [16]). Here we concentrate on a computational aspect from the model to its solution. We will show that the integral representation by means of the Kurzweil-Stieltjes integral is a very natural approach, which coincides with the classical integral representation in the case of DDE's with continuous initial functions (see [21, Lemma 2.1]). As applications, we will discuss the step method and the problem of vanishing points of discontinuity for solutions (studied in [4, 5, 38], for instance).

The paper is recommended for all readers interested on DDE's and is organized as follows. The next two sections are devoted to recall fundamental concepts and basic facts, respectively, about the space of regulated functions and on Henstock-Kurzweil and Kurzweil-Stieltjes integrals. In the fourth section we introduce the notion of regulated solutions and we take into consideration other notions of solutions from the classical theory and some generalized notions of solution. Then we give the integral representation for regulated solutions and we show that classical and Carathéodory solutions as well as piecewise continuous and natural solutions are special cases of regulated ones.

Adapting the step method for the new integral representation we allow its use for problems with continuous and discontinuous initial functions $\phi$ and we prove also some existence theorems. For discontinuous solutions we solve the problem of vanishing discontinuity points (cf. [12]). In the last section we study population dynamics for discontinuous past states (initial functions). The paper is supplemented by explanatory examples.

\section{Regulated FunCtions.}

We denote by $\mathbb{R}$ the set of all real numbers. Given $a, b \in \mathbb{R}, a<b$, we denote by $C([a, b], \mathbb{R})$, $P C([a, b], \mathbb{R}), C^{1}([a, b], \mathbb{R})$ and $A C([a, b], \mathbb{R})$ the space of all continuous, piecewise continuous (cf. $[7,8])$, continuously differentiable and absolutely continuous real-valued functions $u$ defined on $[a, b]$, 
respectively. A function $u:[a, b] \rightarrow \mathbb{R}$ is said to be regulated if there exist finite the right $u\left(t^{+}\right)$and left $u\left(s^{-}\right)$limits for every points $t \in[a, b)$ and $s \in(a, b]$. The Banach space $G([a, b], \mathbb{R})$ consists of all regulated real-valued functions defined on $[a, b]$ (see [23]) equipped with the supremum-norm. Every regulated function is bounded, has a countable set of discontinuities and is the limit of a uniformly convergent sequence of step functions. Clearly $C([a, b], \mathbb{R}) \subset G([a, b], \mathbb{R})$. We recall that a function $g:[a, b] \rightarrow \mathbb{R}$ is said to be of bounded variation if $\operatorname{Var}_{a}^{b} g<\infty$, where the variation $\operatorname{Var}_{a}^{b} g$ is defined by $\operatorname{Var}_{a}^{b} g=\sup \left\{\sum_{i=1}^{p}\left|g\left(t_{i}\right)-g\left(t_{i-1}\right)\right|: a=t_{0} \leq t_{1} \leq \cdots \leq t_{p}=b\right\}$. The Banach space $B V([a, b], \mathbb{R})$ is the space of all functions of bounded variation equipped with the norm $\|g\|_{B V}:=|g(a)|+\operatorname{Var}_{a}^{b} g$. We have $B V([a, b], \mathbb{R}) \subset G([a, b], \mathbb{R})$.

It is well known that a function $x$ of bounded variation can be decomposed as the sum of a continuous function of bounded variation $x_{c}$ and a break function $x_{d}$. Precisely, if $\left\{t_{k}: k \in \mathbb{N}\right\}$, where $\left(t_{k}\right)$ is assumed below to be ordered as increasing, denotes the set of discontinuity points then

$$
x_{d}(t)=\sum_{k=1}^{\infty}\left[\left(x\left(t_{k}\right)-x\left(t_{k}^{-}\right)\right) \chi_{\left[t_{k}, b\right]}+\left(x\left(t_{k}^{+}\right)-x\left(t_{k}\right)\right) \chi_{\left(t_{k}, b\right]}\right] \quad \text { on }[a, b],
$$

where we set $x\left(a^{-}\right)=x(a)$ and $x\left(b^{+}\right)=x(b)$, and $x_{c}=x-x_{d} \in C([a, b], \mathbb{R})$ (cf. [39]). We refer to $x_{c}$ and $x_{d}$ as the continuous and discrete part of $x$. It is know, that $\operatorname{Var}([a, b], x)=\operatorname{Var}\left([a, b], x_{c}\right)+$ $\operatorname{Var}\left([a, b], x_{d}\right)$. Throughout we will deal with the subspace $Z_{G}$ of $G([a, b], \mathbb{R})$ consisting of regulated functions having finite sum of jumps, i.e.

$$
\sum_{t_{k} \in D_{x}}\left[\left|x\left(t_{k}\right)-x\left(t_{k}^{-}\right)\right|+\left|x\left(t_{k}^{+}\right)-x\left(t_{k}\right)\right|\right]<\infty,
$$

where $D_{x}$ denotes the set of discontinuity points of $x$ (so is at most countable).

Proposition 2.1. Any function $x \in Z_{G}$ is representable in the form $x=x_{c}+x_{d}$, where $x_{d}$ is the function, of bounded variation, defined by (2) and $x_{c}=x-x_{d} \in C([a, b], \mathbb{R})$.

Note that $B V([a, b], \mathbb{R}) \subset Z_{G}$ and $C([a, b], \mathbb{R})$ is the subspace of $G([a, b], \mathbb{R})$ consisting of functions having vanishing discrete parts. This class of functions will form a general space consisting of possible initial functions for considered DDE's.

Let $f:[a, b] \times \mathbb{R} \rightarrow \mathbb{R}$, then the Nemytskii superposition operator $S_{f}: \mathbb{R}^{[a, b]} \rightarrow \mathbb{R}^{[a, b]}$ is defined by the formula $S_{f}(x)(t)=f(t, x(t))$. It is one of the most important nonlinear operators in the theory of differential and integral equations. Properties of the Nemytskii operator have been studied in different function spaces (cf. [1]), here we summarize some properties of the operator in the space $G([a, b], \mathbb{R})$. The following useful theorem is proved by Michalak $[27]$.

Theorem 2.1. ([28, Proposition 2.2]) The operator $S_{f}$ maps $G([a, b], \mathbb{R})$ into itself if and only if the function $f$ has the following properties:

(1) the limit $\lim _{[a, s) \times \mathbb{R} \ni(u, y) \rightarrow(s, x)} f(u, y)$ exists for every $(s, x) \in(a, b] \times \mathbb{R}$,

(2) the $\operatorname{limit}_{\lim }(t, b] \times \mathbb{R} \ni(u, y) \rightarrow(s, x) f(u, y)$ exists for every $(t, x) \in[a, b) \times \mathbb{R}$.

In particular, it implies that if $f(t, x(t))=f(x(t))$ then the (autonomous superposition) operator $S_{f}$ maps $G([a, b], \mathbb{R})$ into itself if and only if $f$ is continuous (see [27, Corollary 3.6]).

Corollary 2.1. ([2, Theorem 2.3]) Suppose that the function $f(\cdot, u)$ is regulated on $[a, b]$ for all $u \in \mathbb{R}$, and the function $f(t, \cdot)$ is continuous on $\mathbb{R}$, uniformly with respect to $t \in[a, b]$. Then the operator $S_{f}$ maps $G([a, b], \mathbb{R})$ into itself and is (norm) bounded. 


\section{KurzWEIL-Stieltues integral.}

The Kurzweil-Stieltjes integral will be used in the new integral representation formula for discontinuous solutions of DDE's. Here we collect some important properties of the integral.

A partition of $[a, b]$ is a finite collection $P=\left\{\left(\left[t_{i-1}, t_{i}\right], \xi_{i}\right): i=1,2, \cdots, p\right\}$ where $\left[t_{i-1}, t_{i}\right]$ are non-overlapping intervals in $[a, b], \xi_{i} \in\left[t_{i-1}, t_{i}\right]$ and $\bigcup_{i=1}^{p}\left[t_{i-1}, t_{i}\right]=[a, b]$. A positive function $\delta$ on $[a, b]$ is called a gauge on $[a, b]$. Given a gauge $\delta$ on $[a, b]$, a partition $P=\left\{\left(\left[t_{i-1}, t_{i}\right], \xi_{i}\right), i=\right.$ $1,2, \cdots, p\}$ of $[a, b]$ is called $\delta$-fine if $\left[t_{i-1}, t_{i}\right] \subset\left(\xi_{i}-\delta\left(\xi_{i}\right), \xi_{i}+\delta\left(\xi_{i}\right)\right)$ for $i=1, \ldots, p$.

Definition 3.1. A function $f:[a, b] \rightarrow \mathbb{R}$ is said to be Kurzweil-Stieltjes integrable with respect to $g:[a, b] \rightarrow \mathbb{R}$ on $[a, b]$ (shortly, KS-integrable) if there exists a function denoted by $(K S) \int_{a} f(s) d g(s):[a, b] \rightarrow \mathbb{R}$ such that, for every $\varepsilon>0$, there is a gauge $\delta_{\varepsilon}$ on $[a, b]$ with

$$
\sum_{i=1}^{p}\left\|f\left(\xi_{i}\right)\left(g\left(t_{i}\right)-g\left(t_{i-1}\right)\right)-\left((\mathrm{KS}) \int_{a}^{t_{i}} f(s) d g(s)-(\mathrm{KS}) \int_{a}^{t_{i-1}} f(s) d g(s)\right)\right\|<\varepsilon
$$

for every $\delta_{\varepsilon}$-fine partition $\left\{\left(\left[t_{i-1}, t_{i}\right], \xi_{i}\right): i=1, \ldots, p\right\}$ of $[a, b]$.

The $K S$-integrability is preserved on all sub-intervals of $[a, b]$. The function $t \mapsto(K S) \int_{a}^{t} f(s) d g(s)$ is called the $K S$-primitive of $f$ with respect to $g$ on $[a, b]$, and the $K S$-integral will be denoted by $(K S) \int_{a}^{b} f(s) d g(s)$ or simply by $(K S) \int_{a}^{b} f d g$ (we refer to [33] or [39]). We note that in the case $g(t)=t$ we have the usual Henstock-Kurzweil integral (see [19, 30, 35]).

It is well-known that if $g$ is of bounded variation the $K S$-integral $\int_{a}^{b} f d g$ exists for any regulated function $f$ (or conversely) ([35, Lemma 4.16]) and $\left|(K S) \int_{a}^{b} f d g\right| \leq\|f\|_{\infty}\left(\operatorname{Var}_{a}^{b} g\right)$. Moreover, we have

Proposition 3.1. ([40, Theorem 2.15]) Let $f:[a, b] \rightarrow \mathbb{R}$ be of bounded variation and $g:[a, b] \rightarrow \mathbb{R}$ be regulated. Then both the integrals $(K S) \int_{a}^{b} f d g$ and $(K S) \int_{a}^{b} g d f$ exist and

$$
(K S) \int_{a}^{b} f d g+(K S) \int_{a}^{b} g d f=f(b) g(b)-f(a) g(a)+\sum_{a \leq t \leq b}\left[\Delta^{-} f(t) \Delta^{-} g(t)-\Delta^{+} f(t) \Delta^{+} g(t)\right]
$$

where $\Delta^{+} f(t)=f(t+)-f(t)$ and $\Delta^{-} f(t)=f(t)-f(t-)$.

The following result implies that, under some natural assumptions, the solutions of (1) are regulated functions.

Proposition 3.2. ([14, Theorem 2.2], [39, Proposition 2.3.16.]) Let $g:[a, b] \rightarrow \mathbb{R}$ and $f:[a, b] \rightarrow$ $\mathbb{R}$ be such that the Kurzweil-Stieltjes $(K S) \int_{a}^{b} f(s) d g(s)$ exists. If $g$ is regulated, then so is the primitive $h:[a, b] \rightarrow \mathbb{R}, h(t)=(K S) \int_{a}^{t} f(s) d g(s)$ and for every $t \in[a, b]$,

$$
\Delta^{+} h(t)=h\left(t^{+}\right)-h(t)=f(t)\left[g\left(t^{+}\right)-g(t)\right] \quad \text { and } \quad \Delta^{-}(t)=h(t)-h\left(t^{-}\right)=f(t)\left[g(t)-g\left(t^{-}\right)\right] .
$$

Moreover, if $f$ is bounded and $g$ is of bounded variation, then $h$ is of bounded variation.

In contrast to the case of the Lebesgue integral the primitive $t \rightarrow(H K) \int_{a}^{t} g(s) d s$ is not absolutely continuous. However, such a kind of integrals allows to integrate arbitrary derivative. Recall, that any $A C G_{*}$ function is differentiable a.e. (for basic notions we refer the reader to [32], it contains a survey about generalized notions of absolute continuity of functions), we have the following characterization: 
Proposition 3.3. ([19, Theorem 9.17]) A function $f:[a, b] \rightarrow \mathbb{R}$ is Henstock-Kurzweil integrable on $[a, b]$ if and only if there exists an $A C G_{*}$ function $F$, given by $F(t)=(H K) \int_{a}^{t} f(s) d s$ on $[a, b]$ such that $F^{\prime}(t)=f(t)$ a.e. on $[a, b]$.

In view of Proposition 3.2 we are unable to expect such a nice property for the $K S$-integral. A problem of differentiability is discussed in details in [31].

\section{Delay-differential equations.}

Let now consider problem (1):

$$
\begin{aligned}
x^{\prime}(t) & =f(t, x(t-\tau)) \\
x(t) & =\phi(t) \quad \text { for } t \in[-\tau, 0] .
\end{aligned}
$$

Any solution $x(t)$ of (1) derives its properties from $\phi$ and $f$. Assume $\phi \in G([-\tau, 0], \mathbb{R})$. We expect that all the discontinuity points of $\phi$ will be then propagated in $[0, T]$ as points of discontinuity of the solution $x(t)$. This paper is based on the following definition of solution.

Definition 4.1. A function $x:[-\tau, T] \rightarrow \mathbb{R}$ is called a regulated solution for the problem (1) on $[-\tau, T]$ provided it is a regulated function, it coincides with $\phi$ on $[-\tau, 0]$, is a.e. differentiable on $[0, T]$ and its derivative satisfies the equation $x^{\prime}(t)=f(t, x(t-\tau))$ a.e. on this interval.

In the sequel of this section we will present a short comparison of the above definition with the notions of solution from the classical theory in the case of a continuous initial function $\phi$, and other approaches to define a generalized notion of solution of (1) in the case of a discontinuous $\phi$. In particular, the definition of a regulated solution, together with the integral representation of the problem by means of $K S$-integral, generalizes all these approaches. We will try to justify here that the above definition is the best choice for the study of DDE's and can be used to improve the method of steps.

Considering the case of a continuous function $\phi$, from the classical theory usually a solution is required to be either continuously differentiable or absolutely continuous. Let us recall that a function $x:[-\tau, T] \rightarrow \mathbb{R}$ is called a classical solution for the problem (1) if it is continuous on $[-\tau, 0]$ and it is continuously differentiable on $[0, T]$, its derivative satisfies the equation $x^{\prime}(t)=f(t, x(t-\tau))$ on $[0, T]$ and it coincides with $\phi$ on $[-\tau, 0]$; while $x:[-\tau, T] \rightarrow \mathbb{R}$ is called a Carathéodory solution for the problem (1) if it is continuous on $[-\tau, 0]$ and it is absolutely continuous on $[0, T]$, its derivative satisfies the equation $x^{\prime}(t)=f(t, x(t-\tau))$ a.e on $[0, T]$, and it coincides with $\phi$ on $[-\tau, 0]$.

The definition of regulated solution is a natural extension of both the notions of classical and Carathéodory solution, indeed $C^{1}([-\tau, T], \mathbb{R}) \subset A C([-\tau, T], \mathbb{R}) \subset C([-\tau, T], \mathbb{R}) \subset G([-\tau, T], \mathbb{R})$. Moreover, due to the definition, the assumptions on the operator $S_{f}$ and the linear integral operator $H$ will be less restrictive.

The cases of classical and Carathéodory solution are related to the case when $f$ is, respectively, jointly continuous or a Carathéodory function. Usually such solutions are presented in a very convenient integral form (cf. [21, 22], for instance):

$$
x(t)=\phi(0)+\int_{0}^{t} f(s, x(s-\tau)) d s \quad \text { for } t \geq 0 .
$$

For a brief discussion for DDE's we refer to [4] and much more detailed for oDE's to [13]. Now we look at the attempts to define a generalized notion of a solution for (1). Typically in the case of Carathéodory type (see [5] and [8]) solutions the differentiability is expected except at the points $t=n \tau, n=0,1,2, \ldots$ (cf. the step method). One needs to fix the properties of solutions in such 
points: usually, it is assumed that the solution should be right-continuous and right-differentiable. We point out that the use of the integral representation with the $K S$-integral allows us to control the properties of solutions at those points, taking into account the properties of $\phi$. Note that the discontinuity of $\phi$ implies, that we are unable to find classical solutions, because for any point $t_{0}$ of discontinuity of $\phi$ the derivative $x^{\prime}(t)$ of a solution $x(t)$ cannot exist at time $t=t_{0}+\tau$ (as claimed in $[8$, Lemma 3.2], for instance).

If a delay differential problem, observed in the past, has some discontinuity points ( $\phi$ is discontinuous), then we can expect that the same property should be valid in the future (so-called breaking points, in this paper they are of the form $\left\{\xi_{k}+n \tau: n=1,2, \ldots\right\}$, where $\left(\xi_{k}\right)$ are discontinuity points of $\phi$ ). We point out that, according to Definition 4.1, the negligible set on which the derivative of a regulated solution does not exist may consist of two kind of points: breaking points dependent on $\phi$ and some points of continuity of $x$ in which $x$ has no derivative (points of discontinuity of $f$ ). But when studying discontinuous solutions for differential problem the situation is much more complicated than presented above (see [20, Section 2.2], for an interesting discussion). As clarified in [41, Chapter 5] we have a few different approaches. For the case of DDE's with discontinuous initial function $\phi$ the most appropriate choice seems to be the use of the definition which coincides with Carathéodory solutions for continuous $\phi$ (cf. [8, Lemma 3.2]). An interesting discussion about the notion of a solution for DDE's can be found in [3, Section 1.4].

We recall the definition from [4] and [5] for continuous $f$ and for right-continuous $\phi$ with finite number points of discontinuity (adapted to the case of our problem), which is used to study discontinuous solutions for (1), but it is closer to the notion of solutions for impulsive differential equations (see also [7]).

Definition 4.2. ([4, Definition 3.14], [5, Definition 2.7]) A function $x:[-\tau, T] \rightarrow \mathbb{R}$ is called a natural solution for the problem (1) on $[-\tau, T]$ provided it is right-continuous and satisfies for all $t \in[0, T]$ the equation (1) with the derivatives being taken as right-hand derivatives.

The function $f$ is supposed to be continuous, so the integral, in the integral representation, is taken in the Riemann sense. However, if we don't need to control the (right-) differentiability (as in [4]), then the above definition can serve as a Carathéodory solution too (the differentiability almost everywhere). The above definition suggests to use the integral representation instead of the original one and we will use this idea by presenting the integral representation for regulated solutions. If the points of the future discontinuity are known, then the following definition is used:

Definition 4.3. ([8,38]) A function $x:[-\tau, T] \rightarrow \mathbb{R}$ is called piecewise continuous solution for (1) if it is continuous and satisfies (1) (either everywhere [38] or almost everywhere [8]) on some intervals $\left[t_{k}, t_{k+1}\right) \subset[0, T]$ for some prescribed points $\left(t_{k}\right)(k=1,2, \ldots N)$, possibly dependent on a piecewise continuous initial function $\phi$.

In fact, it is a solution for the set of problems

$$
x^{\prime}(t)=f_{k}(t, x(t-\tau)), \quad x\left(t-t_{k+1}\right)=g_{k}\left(x\left(t-t_{k+1}\right)\right),
$$

where $t \in\left[t_{k}, t_{k+1}\right), k=1,2, \ldots, N$ and $f_{k}, g_{k}$ are sufficiently well-defined. Note that the space of piecewise continuous functions also forms a proper subspace of the space of regulated functions, so it is also a special case of our Definition 4.1 (see also [25, Chapter 2.2]).

Sometimes, functional-differential problems for which piecewise continuous solutions are expected are formulated directly as impulsive delay differential equations (cf. [7]). So the set of solutions is in the space $P C([-\tau, T], \mathbb{R})$ and the initial function is also piecewise continuous. It is connected again with integral problems with the Henstock-Kurzweil integral as observed in [17], but impulsive 
delay differential equations have separated description of the points of discontinuity and jumps and this is not object of our study (cf. [15] for recent results). However, due to the fact that $P C([-\tau, T], \mathbb{R}) \subset G([-\tau, T], \mathbb{R})$, such an approach is close, in some sense, to ours. As claimed in $[3$, Theorem 2.1] any Carathéodory solution is also a piecewise continuous one.

\section{INTEGRAL REPRESENTATION FOR SOLUTIONS OF (1).}

The problem of the existence of an integral representation formula for discontinuous solutions was observed and discussed in [4]. It is worthwhile to note that for natural solutions it was investigated under very restrictive assumptions on $f$ (see [4, Lemma 3.13]), moreover the initial function $\phi$ was assumed to be right-continuous with a finite number of points of discontinuity having bounded jumps (see [4, Assumption 3.2]). We observe that such a class of functions is contained in the space of regulated functions (even in the subspace of the so-called "cádlág" functions).

Denote by $Z_{G([-\tau, 0], \mathbb{R})}$ the subspace of $G([-\tau, 0], \mathbb{R})$ consisting of regulated functions having finite sum of their jumps, i.e. their discrete parts are of bounded variation (see Section 2). We will give a proper equivalent integral representation for (1) in the more general case in which $\phi \in Z_{G([-\tau, 0], \mathbb{R})}$. Throughout we will assume that the operator $S_{f}$ maps the space $G([-\tau, T], \mathbb{R})$ into itself (cf. Theorem 2.1 for the necessary and sufficient conditions). We need to construct

a function $g$ associated with the initial function $\phi$, which will keep all the information about its discontinuity points and then we define an integral form of solutions by means of the $K S$-integral. This approach will allow to prolong discontinuity points in a method of steps and, of course, to keep all the advantages of integral representation of solutions.

Definition 5.1. Assume that $\phi \in Z_{G([-\tau, 0], \mathbb{R})}$. Let $t \in I_{m}=(m \tau,(m+1) \tau)$ for some $m \in \mathbb{N}$ for which $(m+1) \tau \leq T$, then we define a function $g:[0, T] \rightarrow \mathbb{R}$ :

$$
g(t)=(t-m \tau)+\sum_{y<t-(m+1) \tau} \Delta^{-} \phi(y)+\sum_{y \leq t-(m+1) \tau} \Delta^{+} \phi(y),
$$

and if $t$ is one of the endpoints of any interval $I_{m}$ we put the left limits. We call $g$ the associated function with $\phi$. If necessary, we can put also $g(t)=\phi(t)$ for $t \in[-\tau, 0]$ and $g(t)=0$ for $t>T$.

Lemma 5.1. (cf. [34, Proposition 15]) Assume that the function $\phi:[-\tau, 0] \rightarrow \mathbb{R}$ is regulated and let $g$ the associated function with $\phi$. Then

i) The function $g$ is regulated and if $\phi \in Z_{G([-\tau, 0], \mathbb{R})}$, then $g \in B V([0, T], \mathbb{R})$.

ii) Let $\phi \in Z_{G([-\tau, 0], \mathbb{R})}$. Then $\Delta^{-} g(t)=\Delta^{-} \phi(t-(m+1) \tau)$ and $\Delta^{+} g(t)=\Delta^{+} \phi(t-(m+1) \tau)$ for $t \in(m \tau,(m+1) \tau), m \in \mathbb{N}$.

iii) For any regulated function $h:[-\tau, T] \rightarrow \mathbb{R}$ the integral $(K S) \int_{-\tau}^{T} h(s) d g(s)$ is well-defined and its KS-primitive is a regulated function on $[-\tau, T]$.

Proof. i) Clearly $g$ is regulated function. Put $M \geq 1$ be such that $T \leq M \cdot \tau<T+1$. If $\phi$ has its discrete part of bounded variation on $[-\tau, 0]$, then by the definition of the discrete part

$$
\operatorname{Var}_{0}^{T} g \leq(M+1)+\operatorname{Var}_{-\tau}^{0} \phi_{d}<\infty .
$$

ii) Let $t \in(m \tau,(m+1) \tau)$ for some $m \in \mathbb{N}$ and take a sequence $t_{k} \rightarrow t, t_{k}<t$. Note, that by definition, $t$ is a point of continuity of $g$ if and only if both $\Delta^{-} \phi(t-(m+1) \tau)=0$ and $\Delta^{+} \phi(t-(m+1) \tau)=0$, so the thesis holds true. 
Suppose now, that $t$ is such that $t-(m+1) \tau$ is a point of discontinuity of $\phi$. Then

$$
\begin{aligned}
\left|g\left(t_{k}\right)-g(t)\right| & =\mid\left(t_{k}-m \tau\right)+\sum_{y<t_{k}-(m+1) \tau} \Delta^{-} \phi(y)+\sum_{y \leq t-(m+1) \tau} \Delta^{+} \phi(y) \\
& -(t-m \tau)-\sum_{y \leq t-(m+1) \tau} \Delta^{-} \phi(y)-\sum_{y \leq t-(m+1) \tau} \Delta^{+} \phi(y) \mid \\
& \leq\left|t_{k}-t\right|+\sum_{t_{k}-(m+1) \tau<y \leq t-(m+1) \tau}\left|\Delta^{-} \phi(y)\right|+\sum_{t_{k}-(m+1) \tau<y \leq t-(m+1) \tau}\left|\Delta^{+} \phi(y)\right| .
\end{aligned}
$$

Clearly, $\left|t_{k}-t\right| \rightarrow 0$ as $k \rightarrow \infty$. If $t-(m+1) \tau$ is not an accumulation point for the set of all discontinuity points of $\phi$, then for sufficiently big $k$ there is no right discontinuity points in the interval $\left(t_{k}, t\right)$, then $\sum_{t_{k}-(m+1) \tau<y<t-(m+1) \tau}\left|\Delta^{+} \phi(y)\right|=0$. In this case $t$ is the unique point of left discontinuity in this subinterval and then $\sum_{t_{k}-(m+1) \tau<y \leq t-(m+1) \tau}\left|\Delta^{-} \phi(y)\right|=\Delta^{-} \phi(t-(m+1) \tau)$.

Consider now the case, when $t-(m+1) \tau$ is an accumulation point for the set of all discontinuity points of $\phi$. As this function is regulated, it should have limits at $t$. Thus the set $\left\{t \in[-\tau, 0]:|\phi(t)-\phi(t-)|>\frac{1}{k}\right\}$ is finite and consequently $\Delta^{-} \phi\left(t_{k}-(m+1) \tau\right) \rightarrow 0$ as $k \rightarrow \infty$. As $\phi \in Z_{G([-\tau, 0], \mathbb{R})}$, then for $k$ sufficiently big $\sum_{t_{k}-(m+1) \tau<y \leq t-(m+1) \tau}\left|\Delta^{-} \phi(y)\right|<\frac{1}{k}$. Again $\sum_{t_{k}-(m+1) \tau<y \leq t-(m+1) \tau}\left|\Delta^{+} \phi(y)\right|=0$.

Whence $\left|g\left(t_{k}\right)-g(t)\right| \leq\left|t_{k}-t\right|+\left|\Delta^{-} \phi(t-(m+1) \tau)\right|+\frac{1}{k} \rightarrow\left|\Delta^{-} \phi(t-(m+1) \tau)\right|$ as $k \rightarrow \infty$.

Hence for any $t \in[0, T]: \Delta^{-} g(t)=\Delta^{-} \phi(t-(m+1) \tau)$. For the right limit we have similar estimations.

iii) Due to i) $g$ is of bounded variation. If $h$ is regulated, then the integral $(K S) \int_{0}^{t} h(s) d g(s)$ exists and is a regulated function too. Thus it is an immediate consequence of Proposition 3.2.

Observe that if $\phi$ is a continuous function then the discrete part of the associated function $g$ vanishes, so we get $d g(s)=d s$.

Consider now the following integral equation with the $K S$-integral:

$$
x(t)=\phi(0)+(K S) \int_{0}^{t} f(s, x(s-\tau)) d g(s) \text { for } t \in[0, T],
$$

with $x(t)=\phi(t)$ for $t \in[-\tau, 0], \phi:[-\tau, 0] \rightarrow \mathbb{R}$.

We have the following integral representation theorem (cf. [21, Lemma 2.1] for the case of continuous $\phi$ and $f$ ):

Theorem 5.1. Assume, that $\phi \in Z_{G([-\tau, 0], \mathbb{R})}$ and $f:[-\tau, T] \times G([-\tau, T], \mathbb{R}) \rightarrow \mathbb{R}$ is such that the Nemytskii operator $S_{f}$ maps the space $G([-\tau, T], \mathbb{R})$ into itself. Then $x$ is a regulated solution for (1) if and only if it is regulated function satisfying the integral equation (3), where the function $g$ is the associate function with $\phi$.

Proof. Let $\phi \in Z_{G([-\tau, 0], \mathbb{R})}$. Denote by $N$ its set of discontinuity points. We will not expect differentiability for solutions on the set $\{N+k \tau: k=0,1,2, \ldots\}$ (possibly this set can be empty), which is at most a countable set. Let $g$ be the associated function with $\phi$.

I. First assume that $x$ is a regulated solution for (1). Then $x$ is a.e. differentiable on $[-\tau, T]$ and its derivative satisfies the equation $x^{\prime}(t)=f(t, x(t-\tau))$ a.e. on $[-\tau, T]$. Since the derivative of $x$ may not exists in the set $\{N+k \tau: k=0,1,2, \ldots\}$ and for $K S$-integrability we need to have a function everywhere defined, we can put $x^{\prime}(t)=0$ at these points. Clearly $x(\cdot-\tau)$ is also regulated, 
by our assumption on the Nemytskii superposition operator we have that the function $f(\cdot, x(\cdot-\tau))$ is regulated and, by (1), the same is true for $x^{\prime}$.

Since the function $\phi$ is regulated and the mapping $s \mapsto s-\tau$ strictly increasing, then the composition $\phi(\cdot-\tau)$ is regulated too, as a consequence of the Sierpiński characterization of regulated functions ([37]). Then by Lemma 5.1 iii) the integral $(K S) \int_{0}^{t} f(s, x(s-\tau)) d g(s)$ is well-defined. It means that we are able to $K S$-integrate both sides of $x^{\prime}(t)=f(t, x(t-\tau))$ with respect to $g$,

$$
(K S) \int_{0}^{t} x^{\prime}(s) d g(s)=(K S) \int_{0}^{t} f(s, x(s-\tau)) d g(s) .
$$

Consequently, by Proposition 3.2 (as $x^{\prime}(t)=0$ at the point of its discontinuity) we get:

$$
\begin{aligned}
x(t)-x(0) & =(K S) \int_{0}^{t} f(s, x(s-\tau)) d g(s) \\
x(t)-\phi(0) & =(K S) \int_{0}^{t} f(s, x(s-\tau)) d g(s) \\
x(t) & =\phi(0)+(K S) \int_{0}^{t} f(s, x(s-\tau)) d g(s) .
\end{aligned}
$$

Thus $x$ is a solution for (3).

II. Now let $x$ be a regulated function satisfying (3). Then, from the hypothesis on $f$, for any $t \in[0, T]$ the integral $y(t)=(K S) \int_{0}^{t} f(s, x(s-\tau)) d g(s)$ exists and by Proposition $3.3 y$ is an $A C G_{*}$ function, whence differentiable a.e., say outside the null set $N_{1}$ with $y^{\prime}(t)=f(t, x(t-\tau))$ a.e. on $[0, T]$. Moreover by Proposition 3.2, $y$ is a regulated function.

If we differentiate both sides of (3) at a common point of differentiability $t$ (the set with a full measure, i.e. $\left.[0, t] \backslash\left(N_{1} \cup\{N+k \tau: k=0,1,2, \ldots\}\right)\right)$, we get

$$
x^{\prime}(t)=\left((K S) \int_{0}^{t} f(s, x(s-\tau)) d g(s)\right)^{\prime}=y^{\prime}(t)
$$

and clearly $x(0)=\phi(0)=y(0)$, so $x(t)=y(t)$. Therefore the regulated function $x$ satisfies $x^{\prime}(t)=f(t, x(t-\tau))$ a.e. on $[0, T]$. Consequently, it is a regulated solution for (1).

Remark 5.1. The regulated solution satisfies the equation a.e., so if we expect the uniqueness of solutions we need to add some rules how to fulfill the values at the points of discontinuity (and values of a derivative at some points). Recall, that definitions for both natural and piecewise solutions contain such conditions. It is important, when we need to study natural solutions and it was discussed in earlier papers $([3,4,5]$, for instance). But the Stieltjes-type integrals are defined everywhere, so the only possibility is to redefine the function $g$.

As the initial function is defined on a closed interval, some regularity conditions for $\phi$ are necessary. It can have jumps at $t=-\tau$ and $t=0$ and we need to have

$$
\begin{aligned}
x(0) & =\phi(0)+(H K) \int_{0}^{0} f(s, x(s-\tau)) d g(s)=\phi(0)+(H K) \int_{0}^{0} f(s, \phi(s-\tau)) d g(s) \\
& =\phi(0)+f(0, \phi(-\tau)) \cdot[g(0+)-g(0)] .
\end{aligned}
$$

It means that $f(0, \phi(-\tau)) \cdot[g(0+)-g(0)]=f(0, \phi(-\tau)) \cdot[\phi(-\tau+)-\phi(-\tau)]$ should be equal to 0 (cf. [22, p. 50]). 
Now, we should compare our result with a classical one when $\phi$ is continuous. In such a case for classical solutions we should have $S_{f}: C([-\tau, T], \mathbb{R}) \rightarrow C([-\tau, T], \mathbb{R})$, which is equivalent with the fact that $f$ is jointly continuous $([1$, Theorem 6.3$])$. Then

Lemma 5.2. For a given $f:[a, b] \times \mathbb{R} \rightarrow \mathbb{R}$ if the operator $S_{f}$ maps $C([a, b], \mathbb{R})$ into itself, then $S_{f}: G([a, b], \mathbb{R}) \rightarrow G([a, b], \mathbb{R})$.

Proof. In view of Theorem 6.3 in [1] the assumption implies, that $f$ is continuous on $[a, b] \times \mathbb{R}$. In particular, it means, that the following limits exist: $\lim _{[a, s) \times \mathbb{R} \ni(u, y) \rightarrow(s, x)} f(u, y)$ for every $(s, x) \in$ $(a, b] \times \mathbb{R}$ and $\lim _{(t, b] \times \mathbb{R} \ni(u, y) \rightarrow(s, x)} f(u, y)$ for every $(t, x) \in[a, b) \times \mathbb{R}$. Moreover, they are equal $f(s, x)$ and $f(t, x)$. Thus the limits are finite and due to Theorem 2.1 the operator $S_{f}$ maps $G([a, b], \mathbb{R})$ into itself.

\section{Step Method.}

The introduced notion of regulated solutions is also appropriate for a classical step method used for solving DDE's. For the case of (discontinuous) natural solutions it was proposed in [4, Section $4.2]$ and here we will present an extension. Suppose, that $\phi \in Z_{G([-\tau, 0], \mathbb{R})}$ and that the Nemytskii (autonomous) superposition operator $S_{f}$ maps $G([-\tau, T], \mathbb{R})$ into itself (for some $T>0$ ). Consider the integral equation

$$
x(t)=x(0)+(K S) \int_{0}^{t} f(s, x(s-\tau)) d g(s) \text { for } t \in[0, T],
$$

with $x(t)=\phi(t)$ for $t \in[-\tau, 0]$.

Step I. Let $t \in[0, \tau]$. Then we have

$$
x(t)=\phi(0)+(K S) \int_{0}^{t} f(s, x(s-\tau)) d g(s) .
$$

As $s-\tau \in[-\tau, 0]$ we get $x(s-\tau)=\phi(s-\tau)$. Thus by using the substitution formula for the $K S$-integral $([30])$ we get

$$
\begin{aligned}
x(t) & =\phi(0)+(K S) \int_{0}^{t} f(s, x(s-\tau)) d g(s) \\
& =\phi(0)+(K S) \int_{-\tau}^{-\tau+t} f(z+\tau, x(z)) d g(z+\tau) \\
& =\phi(0)+(K S) \int_{-\tau}^{-\tau+t} f(z+\tau, \phi(z)) d g(z+\tau) \\
& =\phi(0)+(K S) \int_{0}^{t} f(s, \phi(s-\tau)) d g(s)=: \phi_{1}(t) .
\end{aligned}
$$

We need to show, that the latter integral exists and is regulated. As claimed in the proof of Theorem 5.1 the integral is well-defined. Since the operator $S_{f}$ maps regulated functions into the same class of functions $\phi_{1}$ is regulated on $[0, \tau]$. We extend it to $[-\tau, \tau]$ by assuming that $\phi_{1}(t)=\phi(t)$ on $[-\tau, 0)$.

Step II. Let $t \in[\tau, 2 \tau]$. Again we have

$$
x(t)=\phi(0)+(K S) \int_{0}^{t} f(s, x(s-\tau)) d g(s)=\phi(0)+(K S) \int_{0}^{t} f\left(s, \phi_{1}(s-\tau)\right) d g(s)=: \phi_{2}(t) .
$$


We are able to repeat our above comments about existence and regularity of function $\phi_{2}$, so this function exists and is regulated. Put again $\phi_{2}(t)=\phi_{1}(t)$ on $[-\tau, \tau)$, so we have a regulated solution on $[-\tau, 2 \tau]$.

Step III. By repeating this procedure we are able to extend a solution $x$ as long as the considered integral exists to $[-\tau, T]$ (by induction).

Remark 6.1. Note that if the equation is of the form (a delayed logistic equation with potentially discontinuous initial function, for instance)

$$
\begin{aligned}
x^{\prime}(t) & =f(t, x(t), x(t-\tau)) \\
x(t) & =\phi(t) \quad \text { for } t \in[-\tau, 0],
\end{aligned}
$$

then in a first step we have $x(t)=\phi(0)+(K S) \int_{0}^{t} f(s, x(s), \phi(s-\tau)) d g(s)$ we get usual differential equation $(\phi(\cdot-\tau)$ is the known function), which can be treated as in [41] and under our assumptions it is possible to find a solution by step method too (cf. also [12]).

We are ready to present an existence theorem based on the step method with assumptions presented in terms of considered operators (i.e. assumptions on $S_{f}$ rather than on $f$ ), which is an extension of that presented in classical books and can be even easily adapted to more general problems.

Theorem 6.1. Suppose, that $\phi \in Z_{G([-\tau, 0], \mathbb{R})}$ and that for some $T>0$ the operator $S_{f}$ maps $G([-\tau, T], \mathbb{R})$ into itself. Then there exists a regulated solution for the problem (1) on $[-\tau, T]$.

Proof. As $\phi$ satisfies all assumptions of Lemma 5.1 the associated function $g$ is regulated and has bounded variation. By our assumption, the function $S_{f}(\phi)$ is regulated on $[-\tau, 0]$, so $K S$-integrable on this interval with respect to $g$. Therefore, the step method can be used (as $S_{f}\left(\phi_{k}\right)$ are regulated functions too for $k=1,2, \ldots$, where $\phi_{k}$ are constructed above) to obtain a regulated solution for the integral equation $(3)$ on $[-\tau, T]$. Thus by Theorem 5.1 we obtain a regulated solution for the problem (1) on $[-\tau, T]$.

As in the case of continuous solutions (cf. [22]) we are able to study the case when $T=\infty$ :

Corollary 6.1. Suppose that the assumptions of Theorem 6.1 hold true. If, moreover, the operator $S_{f}$ is bounded, then we can put $T=\infty$ and any regulated solution can be prolonged up to $T=\infty$.

Remark 6.2. It follows from the above consideration that the classical method is valid for the case when $\phi$ (so $g$ too) is of bounded variation and the substitution formula holds true for the $K S$-integral (and the set of discontinuity points need not be necessarily a finite set, cf. [4]). Thus we are able to treat also different delay-differential problems ([22]) including the case of initial functions with possible countable number of the discontinuity points. It is worthwhile to note that, since $S_{f}(x)$ is regulated, it has at most a countable set of discontinuity points, so the proper choice for the step method is the $K S$-integral representation.

Under our assumptions, when $\phi$ is continuous we have more general existence results, due to the fact that we require the Henstock-Kurzweil integrability of the function $S_{f}(x)(\cdot)$. Its primitive is continuous, but not necessarily absolutely continuous, so even in such a case the solution need not to be a Carathéodory solution. Note, that the Lebesgue integrability implies the Henstock-Kurzweil integrability, but the converse need not be true. However, in both cases the primitives are derivable almost everywhere. In view of Lemma 5.1 we get 
Corollary 6.2. If the initial function $\phi$ is continuous, then the regulated solution is a continuous function. If moreover, $S_{f}(x)(\cdot)$ is Lebesgue integrable, then it is absolutely continuous and we get a Carathéodory solution.

Remark 6.3. The converse implication need not be true, so even for a continuous function $\phi$ we have more general results.

Sometimes, instead of the step method, we need to construct a sequence of approximations for a solution of (1). In such a case we can apply the following convergence proposition (cf. [29, Theorem 6.3] or [30, Corollary 3.4]):

Proposition 6.1. Let $g$ be a function of bounded variation on $[0, T]$ and assume that the Nemytskii operator $S_{f}$ generated by a function $f:[0, T] \times \mathbb{R} \rightarrow \mathbb{R}$ satisfying Carathéodory conditions maps $G([0, T], \mathbb{R})$ into itself. Then for any sequence $\left(x_{n}\right) \subset G([0, T], \mathbb{R})$ pointwisely convergent to some $x \in G([0, T], \mathbb{R})$ such that for some $M>0$ we have $\sup _{n}\left\|S_{f}\left(x_{n}\right)\right\|_{\infty} \leq M<\infty$ we get

$$
\lim _{n \rightarrow \infty}(K S) \int_{0}^{t} f\left(s, x_{n}(s)\right) d g(s)=(K S) \int_{0}^{t} f(s, x(s)) d g(s) \text { for } t \in[0, T] .
$$

By assuming that $\phi \in Z_{G}(-\tau, 0)$, let us consider again the problem (1). Put

$$
x_{1}(t)=\varphi(t) \text { for } t \in[-\tau, 0] \text { and } x_{1}(t)=\varphi(0) \text { for } t \in[0, T] .
$$

Then for any $t \in[0, T]$ we can define a sequence of successive approximations:

$$
x_{n+1}(t)=\varphi(0)+(K S) \int_{0}^{t} f\left(s, x_{n}(s-\tau)\right) d g(s), n \geq 1
$$

(see [4, Section 4.4 and Theorem 4.6] for the applicability of the method of iterations for natural solutions). By our assumptions we ensure that all integrals exists and that the functions $x_{n}(n \in \mathbb{N})$ are regulated. Clearly, $x_{2}$ coincides with a function defined as a solution by the step method on a interval $[0, \tau]$ and is constant in $(\tau, T]$. The same property holds true for $x_{n+1}$ on $[0, n \tau]$ and $(n \tau, T]$, respectively. Thus for any finite $T$ we get a solution on a finite number of steps:

Proposition 6.2. Let $g$ be a function of bounded variation on $[0, T]$ and assume that the Nemytskii superposition operator $S_{f}$ generated by a function $f:[0, T] \times \mathbb{R} \rightarrow \mathbb{R}$ satisfying Carathéodory conditions maps $G([0, T], \mathbb{R})$ into itself. Then there exists a regulated solution of (1), which is obtainable by Picard iteration based on (5).

Note, that for the case $T=\infty$ the sequence $\left(x_{n}\right)$ is pointwisely convergent to a solution of (1).

6.1. Problem of vanishing discontinuity points. It is known that the current version of the step procedure is, in some sense "improving". We believe, that there is no reasons to think, that process which was discontinuous in the past should be continuous in the future. Indeed, even if mathematical models are studied with discontinuous initial function (piecewise continuous, for instance as in [38, Section II] or with jumps like in [9]), then the solutions were continuous. However, in the paper mentioned above the necessity of the study for discontinuous solutions is explained and for some special cases a research method is presented.

Unfortunately, it is based on the description of jumps in the future, i.e. for $t_{k}>0, k \in \mathbb{N}$. It means, that we need to consider a sequence of problems

$$
x^{\prime}(t)=f_{k}(t, x(t-\tau)), \quad x\left(t-t_{k+1}\right)=g_{k}\left(x\left(t-t_{k+1}\right)\right),
$$

where $t \in\left[t_{k}, t_{k+1}\right)$ and $f_{k}, g$ are sufficiently well-defined. Such an approach allows to obtain discontinuous solutions, but only in prescribed points with determined for a countable number 
of points transition functions $g_{k}$ (cf. [38, Section III]). In such a case, we need to solve several differential problems (without use of the step method), we are unable to use classical methods and so the assumptions are really strong (like Lipschitz conditions, for instance). Let us emphasize, that in that paper solutions are just piecewise continuous functions and additional property of rightcontinuity is separated from the existence problem. We should note that, as space $P C([-\tau, T], \mathbb{R})$ is a subspace of the space of regulated functions, our method can be also applied in the case considered in [38] with standard (more natural) formulation of the problem.

Now, let us underline that in [4] one of the problems lies in the fact that after each step the solution is more regular than in the previous step. In particular, in a classical approach with the integral, the points of discontinuity vanish after one step. It means, that we are not able to find discontinuous solutions. Since it is not a realistic case, the authors in $[4,5]$ discussed some assumptions allowing to keep the discontinuities for the next step, described all the discontinuity points in the future ([38]).

In the case of a fixed time delay considered here, the constructed function $g$ allows to preserve the points of discontinuity for the next interval in the step method. More detailed theory devoted to study such a problem for DDE's is presented in [12, Section 2]. It is discussed the fact that as $t$ increases, the solution $x$ of (1) becomes smoother than the initial function ([21]) (a discussion is also for derivatives of a solution, but it goes beyond the goal of this work).

Remark 6.4. We should also mention some theoretical approaches, where DDE's are treated as special cases of generalized ordinary differential equations, dynamic or measure differential equations. In contrast to the case considered in this paper, such papers are devoted to unify some earlier theories and not related to computational aspects.

The first case is when solutions are expected to be functions of bounded variation $([18,36]$, for instance). It is a subspace of the space of regulated functions $G([-\tau, T], \mathbb{R})$. As the norm considered in the space of functions of bounded variation is stronger than the supremum-norm, then additional properties of solutions are required (and so additional assumptions). However, the form of the considered problems are closer to impulsive differential equations ( $\phi$ is supposed to be piecewise continuous with finite number of jumps, whence of bounded variation), solutions are obtained as limits of some approximated solutions. It is interesting, despite that integral representation for considered solutions are not in a Henstock-Kurzweil sense, it has separated continuous and discrete parts, so our approach is valid also for this case.

In [16] some DDE's are studied in the space of regulated functions by considering some integral equations with the $K S$-ntegral, but the goal and the methods used there are completely different, indeed derivatives are taken in the distributional sense and the problem is reformulated to measuredifferential equations or generalized differential equations. Here we prefer the direct approach and we study classical DDE's derived directly from some mathematical models.

The next lemma will allow to check if discontinuity points for the $K S$-integral are preserved. It provides an answer to what we need to assume to keep the continuity or differentiability property at $t=0$ (cf. [12]). It is a basis for our computational method allowing to calculated the points of discontinuity separately.

Lemma 6.1. ([35, Lemma 4.23], [35, Theorem 2.34]) Let $f \in G([a, b], \mathbb{R})$ and $g$ be a regulated function with bounded variation. Denote by $D_{g}$ the set of discontinuity points of $g$ in $(a, b)$ and by 
$g_{c}$ the continuous part of $g$. Then

$$
(K S) \int_{a}^{b} f d g=(K S) \int_{a}^{b} f d g_{c}+f(a) \Delta^{+} g(a)+\sum_{d \in D} f(d) \Delta g(d)+f(b) \Delta^{-} g(b) .
$$

We are able to present a result about non-vanishing continuity points for our problem (1).

Theorem 6.2. Under the assumptions of Theorem 6.1 any regulated solution $x$ of (1) has at every interval $[m \tau,(m+1) \tau]$ the set of discontinuity points of the form $N_{m}=\left\{\xi_{k}+m \tau: k \in \mathbb{N}\right\}$, where $N=\left\{\xi_{k}: k \in \mathbb{N}\right\}$ is the set of discontinuity points of $\phi$ with the exception of points $\xi_{m}$ satisfying the condition $f\left(\xi_{m-1}, x\left(\xi_{m-1}-\tau\right)\right)=0$.

It is an immediate consequence of the above Lemma 6.1. If in the above formula (6) for some point $d$ we have $f(d)=0$, then a discontinuity point vanishes, and for $f(d) \neq 0$ it is distributed for the next interval. Let us omit the detailed proof and, instead, we illustrate the idea by the following example.

Example 6.1. As a special case of $(1)$, for $f(t, x)=t \cdot x$, we consider the following problem:

$$
\begin{aligned}
x^{\prime}(t) & =t \cdot x(t-1) \\
x(t) & =\phi(t) \quad \text { for } t \in[-1,0],
\end{aligned}
$$

with

$$
\phi(t)= \begin{cases}t & \text { for } \quad t \in\left[-1,-\frac{2}{3}\right) \\ t+\frac{2}{3} & \text { for } \quad t \in\left[-\frac{2}{3},-\frac{1}{3}\right) \\ t+2 & \text { for } \quad t \in\left[-\frac{1}{3}, 0\right]\end{cases}
$$

Then $\Delta^{-} \phi\left(-\frac{2}{3}\right)=\frac{2}{3}$ and $\Delta^{-} \phi\left(-\frac{1}{3}\right)=\frac{4}{3}$.

Let $g:[0,1] \rightarrow \mathbb{R}$ be the associate function with $\phi$, defined according to Definition 5.1. Then we have $g(t)=t$ for $t \in\left[0, \frac{1}{3}\right), g(t)=t+\Delta^{-} \phi\left(-\frac{2}{3}\right)=t+\frac{2}{3}$ for $t \in\left[\frac{1}{3}, \frac{2}{3}\right)$ and $g(t)=t+\Delta^{-} \phi\left(-\frac{2}{3}\right)+$ $\Delta^{-} \phi\left(-\frac{1}{3}\right)=t+2$ for $t \in\left[\frac{2}{3}, 1\right]$. Clearly, $g_{c}(t)=t$.

We will calculate the solution of $(7)$ on $[-1,1]$. By applying Lemma 5.1, let us consider three cases:

A) Let $t \in\left[0, \frac{1}{3}\right)$. Then

$$
\begin{aligned}
x(t) & =\phi(0)+(K S) \int_{0}^{t} f(s, \phi(s-1)) d g(s) \\
& =\phi(0)+\int_{0}^{t} s \cdot \phi(s-1) d s=2+\int_{0}^{t} s^{2} d s=2+\frac{1}{3} t^{3} .
\end{aligned}
$$

B) Let $t \in\left[\frac{1}{3}, \frac{2}{3}\right)$. Then

$$
\begin{aligned}
x(t) & =\phi(0)+(K S) \int_{0}^{t} f(s, \phi(s-1)) d g(s) \\
& =2+\int_{0}^{t} s \cdot \phi(s-1) d s+f\left(\frac{1}{3}, \phi\left(-\frac{2}{3}\right)\right) \cdot \Delta^{-} g\left(\frac{1}{3}\right) \\
& =2+\int_{0}^{\frac{1}{3}} s \cdot \phi(s-1) d s+\int_{\frac{1}{3}}^{t} s \cdot \phi(s-1) d s+0 \\
& =2+\int_{0}^{\frac{1}{3}} s^{2} d s+\int_{\frac{1}{3}}^{t} s \cdot\left(s+\frac{2}{3}\right) d s=\frac{1}{3} t^{3}+\frac{t^{2}}{2}+\frac{53}{27} .
\end{aligned}
$$


Observe that $\lim _{t \rightarrow \frac{1}{3}^{-}} x(t)=2+\frac{1}{81}$ and $\lim _{t \rightarrow \frac{1}{3}^{+}} x(t)=2+\frac{1}{81}$, therefore the function $x(t)$ is continuous at $t=\frac{1}{3}$.

C) Let $t \in\left[\frac{2}{3}, 1\right]$. Then

$$
\begin{aligned}
x(t) & =\phi(0)+(K S) \int_{0}^{t} f(s, \phi(s-1)) d g(s) \\
& =2+\int_{0}^{t} f(s, \phi(s-1)) d s+f\left(\frac{1}{3}, \phi\left(-\frac{2}{3}\right)\right) \cdot \Delta^{-} g\left(\frac{1}{3}\right)+f\left(\frac{2}{3}, \phi\left(-\frac{1}{3}\right)\right) \cdot \Delta^{-} g\left(\frac{2}{3}\right) \\
& =2+\int_{0}^{t} s \cdot \phi(s-1) d s+0 \cdot \frac{2}{3}+f\left(-\frac{1}{3}, \phi\left(-\frac{1}{3}\right)\right) \cdot 2 \\
& =2+\int_{0}^{\frac{1}{3}} s \cdot \phi(s-1) d s+\int_{\frac{1}{3}}^{\frac{2}{3}} s \cdot \phi(s-1) d s+\int_{\frac{2}{3}}^{t} s \cdot \phi(s-1) d s+f\left(\frac{2}{3}, \phi\left(-\frac{1}{3}\right)\right) \cdot 2 \\
& =2+\int_{0}^{\frac{1}{3}} s^{2} d s+\int_{\frac{1}{3}}^{\frac{2}{3}} s \cdot\left(s+\frac{2}{3}\right) d s+\int_{\frac{2}{3}}^{t} s \cdot(s+2) d s+\frac{2}{3} \cdot \frac{4}{3} \cdot 2 \\
& =\frac{t^{3}}{3}+t^{2}+\frac{31}{9} .
\end{aligned}
$$

Observe now, that $\lim _{t \rightarrow \frac{2}{3}-} x(t)=2+\frac{23}{81}$ and $\lim _{t \rightarrow \frac{2}{3}}+x(t)=2+\frac{161}{81}$, therefore the function $x(t)$ is discontinuous at $t=\frac{2}{3}$.

Finally,

$$
\phi_{1}(t)= \begin{cases}\frac{1}{3} t^{3}+2 & \text { for } t \in\left[0, \frac{1}{3}\right], \\ \frac{1}{3} t^{3}+\frac{t^{2}}{2}+\frac{53}{27} & \text { for } t \in\left[\frac{1}{3}, \frac{2}{3}\right], \\ \frac{1}{3} t^{3}+t^{2}+\frac{31}{9} & \text { for } t \in\left(\frac{2}{3}, 1\right] .\end{cases}
$$

Thus the function $\phi_{1}$ has a discontinuity point only at $t=\frac{2}{3}$ and not at $t=\frac{1}{3}$. Note that in the next steps the situation will be the same.

If we intend to find a solution on the interval $[1,2]$, then the integral form of the problem is the following

$$
x(t)=\phi_{1}(1)+(K S) \int_{1}^{t} f\left(s, \phi_{1}(s-1)\right) d g(s)=\frac{43}{9}+(K S) \int_{1}^{t} s \cdot \phi_{1}(s-1) d g(s) .
$$

Note, that $g$ is defined for the values in this interval and $g(t)=t-1+\sum_{y \leq t-2} \Delta^{-} \phi(y)$.

Let us concentrate now on a point $t_{0}=\frac{4}{3}$. Note that in this case the point $t_{0}-2$ is a discontinuity point (of $\phi$ ) and $t_{0}-1$ is not (see the above formula for $\phi_{1}$ ).

If $1 \leq t<\frac{4}{3}$, then

$$
x(t)=\frac{43}{9}+\int_{1}^{t} s \cdot\left(2+\frac{1}{3} s^{3}\right) d s=\frac{1}{15} t^{5}+t^{2}+\frac{167}{45} .
$$


For $t \in\left[\frac{4}{3}, \frac{5}{3}\right]$ we obtain

$$
\begin{aligned}
x(t) & =\frac{43}{9}+\int_{1}^{t} s \cdot \phi_{1}(s-1) d s+\frac{4}{3} \cdot \phi_{1}\left(\frac{1}{3}\right) \cdot \Delta \phi\left(-\frac{1}{3}\right) \\
& =\frac{43}{9}+\int_{1}^{\frac{4}{3}} s \cdot\left(2+\frac{1}{3} s^{3}\right) d s+\int_{\frac{4}{3}}^{t} s \cdot\left(2-\frac{1}{27}+\frac{1}{3} t^{3}+\frac{t^{2}}{2}\right) d s \\
& +\frac{4}{3} \cdot \phi_{1}\left(\frac{1}{3}\right) \cdot \Delta^{-} \phi\left(-\frac{1}{3}\right) .
\end{aligned}
$$

Thus the discontinuity comes back as the integrand is not zero at the point $t_{0}$.

\section{An extension.}

Till now our approach is presented for retarded differential equations (1). It can be used for the case of the growth of cell modelling $\left(x^{\prime}(t)=\frac{1}{\tau} x(t-\tau)\right.$, cf. [6]), also for the case of discontinuous initial function $\phi$ ([3, Section 1.4] with piecewise continuous solutions). Now, we are able to study population dynamics also for discontinuous past states and we are not restricted to the cases studied earlier.

Let us consider now DDE of the form:

$$
\begin{aligned}
\frac{d}{d t}\{x(t)-x(t-\tau)\} & =f(t, x(t-\tau)) \\
x(t) & =\phi(t) \text { for } t \in[-\tau, 0] .
\end{aligned}
$$

Let $g$ the associated function with $\phi$. Then by integrating the equation we get

$$
(H K) \int_{0}^{t}\left(\frac{d}{d t}\{x(s)-x(s-\tau)\}\right) d s=(K S) \int_{0}^{t} f(s, x(s-\tau)) d g(s)
$$

and whence

$$
x(t)-x(t-\tau)=\phi(0)-\phi(-\tau)+(K S) \int_{0}^{t} f(s, x(s-\tau)) d g(s) .
$$

Again for $t \in[0, \tau]$ we have the integral representation of a solution:

$$
x(t)=\phi(0)-\phi(-\tau)+\phi(t-\tau)+(K S) \int_{0}^{t} f(s, x(s-\tau)) d g(s) .
$$

Clearly, its properties are dependent directly on the properties of $\phi$ as well as on the properties of operators $S_{f}$ and $H$. Therefore, our previous results are applicable in this case.

Note, that if we need to investigate (8) in an explicit form we need to require that there exist derivatives $x^{\prime}(t)$ and for $\tau(t)$ (the existence of a derivative of $x(t)-x(t-\tau)$ does not imply the existence of $x^{\prime}(t)$ ). In [5] (see also [4, Example 2.1] and [4, Section 4.4]) the above problem with $f(t, x)=x$ is investigated:

$$
\begin{aligned}
\frac{d}{d t}\{x(t)-x(t-\tau)\} & =x(t-\tau) \\
x(t) & =\phi(t) \quad \text { for } \quad t \in[-\tau, 0],
\end{aligned}
$$

where the initial function $\phi(t)=t$ for $t<0$ and $\phi(0)=1$. The natural solution $x$ (obtained by the method of steps) is studied. It is discontinuous function, but $x(\cdot)-x(\cdot-\tau)$ is continuous and has a continuous two-sided derivative for all $t>0$. However, it does not imply the continuity of $x(t)$ and $x(t)$ inherits discontinuities, for certain $t \geq 0$, from discontinuities at earlier times, through dependency on $x(t-\tau)$. 
Let us investigate regulated solutions on $[-1,1]$. In this case $g(t)=t$ for $t<0, g(0)=1$ and $g(t)=t+1$ for $t \in(0,1), g(1)=3$. Its integral representation for $t \in[0,1)$ is given by

$$
x(t)=1+1+(t-1)+(K S) \int_{0}^{t} \phi(s-1) d g(s)=t+1+\int_{0}^{t}(s-1) d s=\frac{1}{2} t^{2}+1
$$

and $x(1)=\frac{1}{2}+1+1$. It means that the regulated solution coincides with the natural one consider in [5], but now we are able to apply directly the new integral representation of a solution. Note, that it is the simplest case, when $\phi$ is discontinuous only at the right-endpoint of the interval. Obviously, this solution can be calculated on next intervals by the same manner.

\section{REFERENCES}

[1] J. Appell, P.P. Zabrejko, Nonlinear Superposition Operators, Cambridge Tracts in Mathematics 95, Cambridge University Press, Cambridge, 1990.

[2] W. Aziz, N. Merentes, J.L. Sánchez, A note on the composition of regular functions, J. Anal. Appl. 20 (2014), $1-5$.

[3] Ch.T.H. Baker, G. Bocharov, F.A.R. Rihan, Neutral delay differential equations in the modelling of cell growth, University of Chester, 2008.

[4] Ch.T.H. Baker, P.M. Lumb, On integral equation formulations of a class of evolutionary equations with time-lag, J. Integral Equations Appl. 18 (2006), 227-247.

[5] Ch.T.H. Baker, Ch.A.H. Paul, Discontinuous solutions of neutral delay differential equations, Appl. Numer. Math. 56 (2006), 284-304.

[6] Ch.T.H. Baker, G.A. Bocharov, Computational aspects of time-lag models of Marchuk type that arise in immunology, Russian J. Numer. Anal. Math. Modelling 20 (2005), 247-262.

[7] G. Ballinger, X. Liu, Continuous dependence on initial values for impulsive delay differential equations, Appl. Math. Lett. 17 (2004), 483-490.

[8] G. Ballinger, X. Liu, Existence, uniqueness and boundedness results for impulsive delay differential equations, Appl. Anal. 74 (2000), 71-93.

[9] A. Bellen, N. Guglielmi, Solving neutral delay differential equations with state-dependent delays, J. Comput. Appl. Math. 229 (2009), 350-362.

[10] M. Bohner, A. Peterson, Dynamic Equations on Time Scales: An Introduction with Applications, Springer, Berlin, 2012.

[11] H. Brunner, The numerical analysis of functional integral and integro-differential equations of Volterra type, Acta Numer. 13 (2004), 55-145.

[12] H. Brunner, W.K. Zhang, Primary discontinuities in solutions for delay integro-differential equations, Methods Appl. Anal. 6 (1999), 525-534.

[13] M. Cichon, On solutions of differential equations in Banach spaces, Nonlinear Anal. 60 (2005), 651-667.

[14] M. Federson, R. Bianconi, Linear integral equations of Volterra concerning Henstock integrals, Real Anal. Exchange 25 (1999/00), 389-417.

[15] M. Federson, J.G. Mesquita, A new continuous dependence result for impulsive retarded functional differential equations, Czechoslovak Math. J. 66 (2016), 1-12.

[16] M. Federson, J.G. Mesquita, A. Slavík, Measure functional differential equations and functional dynamic equations on time scales, J. Differential Equations 252 (2012), 3816-3847.

[17] M. Federson, Š. Schwabik, Generalized ODE approach to impulsive retarded functional differential equations, Differential Integral Equations 19 (2006), 1201-1234.

[18] Yu.N. Fetisova, A.N. Sesekin, Discontinuous solutions of differential equations with time delay, WSEAS Trans. Syst. 4 (2005), 487-492.

[19] R.A. Gordon, The Integrals of Lebesgue, Denjoy, Perron and Henstock, AMS, 1994.

[20] N. Guglielmi, E. Hairer, Computing breaking points in implicit delay differential equations, Adv. Comput. Math. 29 (2008), 229-247.

[21] J.K. Hale, Functional Differential Equations, Springer, Berlin, 1971.

[22] J.K. Hale, S.M. Verduyn Lunel, Introduction to Functional Differential Equations, Vol. 99, Springer, Berlin, 2013.

[23] C.S. Hönig, Equations intégrales generalisées et applications, Pub. Math. d'Orsay 5, 1983. 
[24] M. Jarnicki, P. Pflug, Continuous Nowhere Differentiable Functions: the Monsters of Analysis, Springer, Berlin, 2015.

[25] V. Kolmanovskii, A. Myshkis, Applied Theory of Functional Differential Equations, Vol. 85, Springer, Berlin, 2012.

[26] E. Liz, R. Pouso, Existence theory for first order discontinuous functional differential equations, Proc. Amer. Math. Soc. 130 (2002), 3301-3311.

[27] A. Michalak, On superposition operators in spaces of regular and of bounded variation functions, Z. Anal. Anwend. 35 (2016), 285-308.

[28] A. Michalak, On superposition operators in spaces $B V_{\varphi}(0,1)$, J. Math. Anal. Appl. 443 (2016), 1370-1388.

[29] G.A. Monteiro, U.M. Hanung, M. Tvrdý, Bounded convergence theorem for abstract Kurzweil-Stieltjes integral, Monatsh. Math. 180 (2016), 409-434.

[30] G.A. Monteiro, M. Tvrdý, On Kurzweil-Stieltjes integral in a Banach space, Math. Bohem. 137 (2012), 365-381.

[31] R. Pouso, A. Rodríguez, A new unification of continuous, discrete, and impulsive calculus through Stieltjes derivatives, Real Anal. Exchange 40 (2015), 319-354.

[32] S. Saks, Theory of the Integral, IM PAN, Warszawa, 1937.

[33] S̆. Schwabik, Linear operators in the space of regulated functions, Math. Bohem. 117 (1992), 79-92.

[34] Š. Schwabik, Abstract Perron-Stieltjes integral, Math. Bohem. 121 (1996), 425-447.

[35] Š. Schwabik, M. Tvrdý, O. Vejvoda, Differential and Integral Equations: Boundary Value Problems and Adjoints, Academia, Praha and D. Reidel, Dordrecht, 1979.

[36] A.N. Sesekin, Yu.V. Fetisova, Functional differential equations in the space of functions of bounded variation, Proc. Steklov Inst. Math. 269 (2010), 258-265.

[37] W. Sierpiński, Sur une propriété des fonctions qui n'ont que des discontinuités de première espéce, Bull. Math. Soc. Sci. Math. Roumanie 16 (1933), 1-4. (in French)

[38] Y. Sun, A.N. Michel, G. Zhai, Stability of discontinuous retarded functional differential equations with applications, IEEE Trans. Automat. Control 50 (2005), 1090-1105.

[39] M. Tvrdý, Differential and Integral Equations in the Space of Regulated Functions, Mem. Differential Equations Math. Phys. 25 (2002), 1-104.

[40] M. Tvrdý, Regulated functions and the Perron-Stieltjes integral, Časopis pro Pěstování Matematiky 114 (1989), $187-209$.

[41] S.T. Zavalishchin, A.N. Sesekin, Discontinuous Solutions to Ordinary Nonlinear Differential Equations in the Space of Functions of Bounded Variation, in: Dynamic Impulse Systems, Springer, Netherlands, 1997.

Department of Mathematics and Computer Science, University of Palermo, Via Archirafi 34, 90123 PALERmo, Italy

E-mail address: diana.caponetti@unipa.it

Faculty of Mathematics and Computer Science, A. Mickiewicz University, Umultowska 87, 61-614 Poznań, Poland

E-mail address: mcichon@amu.edu.pl

Department of Mathematics and Computer Science, University of Palermo, Via Archirafi 34, 90123 Palermo, Italy

E-mail address: valeria.marraffa@unipa.it 